\title{
ÉTICA Y METODOLOGÍA DE LAS INVESTIGACIONES SOCIOLINGÜÍSTICAS EN LAS COMUNIDADES VULNERABLES
}

\author{
Miroslav Valeš
}

\begin{abstract}
Standard ethical procedures, based on the informed consent, may result hardly applicable in vulnerable communities. In the same way the methodology of research encounters specific problems in these communities. Based on a personal experience gained during the research of Lakhota language revitalization in South Dakota and Shuar language in Ecuador, this paper intends to draw attention to some methodological problems and to the relativity of ethical criteria. Respectful treatment of participants in the research should be the primary preoccupation of any sociolinguist. For this reason, one of the main objectives of this paper is to propose some general rules to be considered when dealing with vulnerable communities.
\end{abstract}

Key words: ethics; sociolinguistic research; informed consent; vulnerable subject; vulnerable community; participant.

Resumen: Los procedimientos éticos estándares, basados en el consentimiento informado, pueden resultar difícilmente aplicables en las comunidades vulnerables. Asimismo, la metodología de la investigación en dichas comunidades se enfrenta con problemas específicos. A partir de la experiencia personal de las investigaciones realizadas con el pueblo lakhota (EE. UU.) y shuar (Ecuador), esta reflexión intenta llamar la atención sobre algunos problemas metodológicos y sobre la relatividad de los criterios éticos. El trato respetuoso hacia los participantes de una investigación debería ser la preocupación fundamental de todo investigador sociolingüístico. Por esta razón, uno de los objetivos de la comunicación consiste en sugerir unas reglas generales que deben ser consideradas al tratar las comunidades vulnerables.

Palabras claves: ética; investigación sociolingüística; consentimiento informado; sujeto vulnerable; comunidad vulnerable; participante.

\section{Introducción}

Las investigaciones sociolingüísticas, al igual que todas las investigaciones que utilizan los sujetos humanos como una fuente de datos, se enfrentan con el dilema de la ética de la investigación. Los principios básicos de las investigaciones que incluyen los sujetos humanos: el respeto, la beneficencia y la justicia están retratados en el «Belmont Report», un documento básico que regula este tipo de investigaciones en los Estados Unidos, con la supervisión de los HRC (Human Research Committee). La herramienta principal que asegura el trato respetuoso de los sujetos involucrados es el consentimiento informado que el participante de la investigación debe expresar explícitamente. Este procedimiento 
asegura que el participante obtiene suficiente información sobre los procedimientos que comprenden su participación y que declara su decisión voluntaria de emprenderlos.

En la mayoría de los países de la Unión Europea los investigadores no están obligados por sus instituciones a aplicar el consentimiento informado y, por consiguiente, esta responsabilidad queda a su libre albedrío. Sin embargo, esto no significa que los aspectos éticos de las investigaciones lingüísticas sean de menor importancia, más aún en las comunidades vulnerables, donde los participantes solo tienen un conocimiento limitado al decidir sobre su voluntad de participar. De hecho, el objetivo de este trabajo es el de acentuar la trascendencia de los temas éticos, ya que el trato respetuoso hacia los participantes de una investigación debería ser la preocupación fundamental de todo investigador.

El siguiente trabajo se fundamenta en una experiencia personal adquirida durante las investigaciones realizadas con los pueblos lakhota (EE. UU.) y shuar (Ecuador). Estas investigaciones revelaron que en las comunidades vulnerables los procedimientos éticos estándares, fundamentados en el consentimiento informado, pueden resultar de difícil aplicación. Además, revelaron diferencias sustanciales entre las dos comunidades, y por consiguiente las consideraciones éticas no siempre son transferibles de una comunidad a la otra. Asimismo, la metodología de la investigación en dichas comunidades se enfrentaba con problemas específicos, los cuales se comentarán a continuación.

\section{Consentimiento informado}

Los procedimientos éticos se fundamentan en el consentimiento informado, un documento en el cual tanto el participante como el investigador firman su conformidad con el proceso de la participación y con el uso de los datos obtenidos. La finalidad principal de este documento es la de asegurar la confidencialidad de los datos y el respeto a la privacidad del participante. Por esta razón, un consentimiento informado incluye: el título del estudio, el contacto al investigador y su institución, la descripción del proyecto, la descripción del procedimiento, el derecho de terminar la participación en cualquier momento, la promesa de confidencialidad de los datos, el acuerdo de la remuneración o su ausencia, el acuerdo con la grabación, el acuerdo con la publicación de los resultados, las firmas y la fecha.

Un documento en forma de papel no es el único formato que puede tener un consentimiento informado. Otra posibilidad es la de grabar el consentimiento del participante en forma audio o audiovisual. El consentimiento grabado abarca los mismos puntos, pero, por contra, tiene el inconveniente de que el participante no cuenta con la posibilidad de contactar al investigador posteriormente, puesto que no se queda con una copia de su dirección de contacto, y tampoco se queda con una prueba tácita de su acuerdo y de los compromisos del investigador. Considerando estas desventajas es preferible utilizar el consentimiento escrito siempre y cuando resulte posible.

La aplicación del consentimiento informado conlleva múltiples dificultades generales. Por ejemplo, el participante debería estar verdaderamente informado del objetivo de la investigación. Ahora bien, si cumplimos esta condición, ni tan siquiera nos vamos a acercar al habla natural del participante. Otro inconveniente lo puede representar el tiempo necesario para leer un consentimiento informado debidamente elaborado. Sin embargo, cómo estas no son exclusivas de las comunidades vulnerables no forman el enfoque principal de este trabajo. 
Ética y metodología de las investigaciones sociolingüísticas en las comunidades vulnerables

\section{Comunidades vulnerables}

Las normas éticas de las investigaciones que incluyen los sujetos humanos prestan especial atención a los «sujetos vulnerables». «Los sujetos vulnerables son aquellos participantes que no tienen la capacidad mental necesaria para entender y procesar la información respecto a su posible participación o que no tienen completa libertad en la decisión de su participación, a causa de influencia de otras personas, por ejemplo en sociedades estrictamente jerárquicas como el ejército. Otros ejemplos de los sujetos vulnerables son los menores, embarazadas, personas marginadas, prisioneros, refugiados, minorías étnicas, los sin techo o los que viven en una situación de extrema pobreza y, en general, todos aquellos que no son capaces de decidir sobre su participación de manera adecuada" (Valeš 2010: 50).

De manera paralela al término «sujeto vulnerable» podemos hablar también de las «comunidades vulnerables» que forman el enfoque principal de nuestras consideraciones. Con esta terminología nos referimos a las comunidades marginalizadas, extremamente pobres, a las tribus que siguen hasta cierto punto su modo original de vida o a los pueblos indígenas en aislamiento voluntario. Es sumamente difícil imaginarse que los miembros de aquellas comunidades puedan tomar una decisión informada respecto a su participación, ya que no saben nada sobre sus derechos de la confidencialidad de los datos personales, entre otros aspectos.

Una vez que decidimos emprender una investigación en una comunidad de estas características, los temas éticos se vuelven considerablemente más complicados. Por ejemplo, a diferencia de la recogida de datos de sujetos menores cuyas decisiones se toman por medio de sus progenitores, en el caso de las comunidades vulnerables nadie tiene el derecho legal de decidir en su lugar.

\section{Consideraciones éticas}

La aplicación del procedimiento fundamental que asegura la ética de las investigaciones lingüísticas - el consentimiento informado - se enfrenta con dificultades inesperadas en las comunidades vulnerables. El primer aspecto que se debe tener en cuenta es que nuestros participantes probablemente desconocen nuestras leyes y normas éticas. Los miembros de estas comunidades no saben nada de aspectos como, por ejemplo, el sistema legal de nuestra sociedad o los derechos sobre la confidencialidad de datos personales. Algunos de ellos ni tan si quiera están alfabetizados y, por consiguiente, es absurdo dejarles firmar un documento cuyo contenido es imposible de descifrar para ello. En estas situaciones podemos conseguir un consentimiento con la participación, pero este consentimiento no va a ser verdaderamente «informado». Johnstone (2000: 42) dice: «In some cases this procedure, informal consent rather than truly informed consent, may be adequate», sugiriendo que en algunos casos el consentimiento informal puede ser adecuado.

La experiencia de la aplicación del consentimiento informado durante la investigación en la comunidad shuar confirma este axioma. Al reclutar posibles participantes en esta comunidad, inevitablemente les explicábamos de manera resumida las condiciones de la participación, mencionando las grabaciones y la remuneración. Los sujetos que consentían su participación naturalmente tomaban la fase inicial por terminada y no veían ninguna razón para volver a hablar de las condiciones, procedimiento o de los riesgos. Para 
su sorpresa el investigador les empezó a explicar detalles sobre la confidencialidad de los datos personales y el uso de las grabaciones para los fines de investigación y no para el «beneficio financiero». En general, al participante se le reveló toda una serie de temas de los cuales no pensaba al dar el consentimiento inicial y los cuales les fueron realmente difíciles de entender. Muchos de los participantes se inquietaban, porque no sabían cómo interpretar esta información nueva. Sin embargo, la finalidad del consentimiento informado no es la de inquietar al posible participante, y por esta razón, gradualmente quitamos todos los temas sensibles de nuestra explicación para que los participantes se sintieran cómodos. De esta forma llegamos a un consentimiento bastante informal, que posiblemente no cumpliera con todos los requisitos de un consentimiento debidamente elaborado, pero que seguramente se ajustaba mejor a las expectativas de los participantes.

En cambio, en la comunidad lakhota, donde los participantes conocen el sistema legal occidental, los participantes no se sorprendían al ver una hoja de consentimiento informado y en su mayoría tranquilamente firmaban con una actitud de comprensión de cumplir con una obligación institucional.

El ejemplo de la aplicación del consentimiento informado enfatiza el hecho de que en las comunidades vulnerables es necesario considerar de manera más profunda el comportamiento ético, y no limitarse a seguir los modelos estándares. De la misma manera, hay que señalar que existen diferencias sustanciales entre las comunidades. Las siguientes cinco reglas generales no cubren de manera exhaustiva las consideraciones éticas en las comunidades vulnerables, más bien intentan sugerir algunos de los aspectos que no se pueden descuidar (Valeš 2010: 53-54):

1. No grabar de manera secreta.

2. Evitar comunidades indígenas poco contactadas.

3. No interferir en la política y/o conflictos locales.

4. No evaluar las situaciones solo con nuestros criterios y opiniones de lo moral y lo ético.

5. Estar dispuesto a ofrecer algo a cambio.

Respecto a la primera regla, las grabaciones secretas son éticamente inaceptables y esto naturalmente sigue siendo válido en todas las situaciones, más aún en las comunidades vulnerables.

En cuanto a la segunda, es preferible que los participantes de un estudio sociolingüístico sean personas que están en contacto con la cultura europea. De esta manera van a compartir, «la misma ética» con el investigador y se evitarán malentendidos. Naturalmente, los derechos de los pueblos indígenas que viven en aislamiento voluntario deben ser respetados y un investigador responsable no se aventura en sus territorios.

La tercera regla puede parecer demasiado obvia, sin embargo, su cumplimiento a veces no es tan sencillo. La misma terminología «política lingüística» alude a la relación entre política y lingüística. Los lazos entre la política y lingüística se hacen especialmente intensos en caso de las comunidades multilingües con una lengua amenazada. En estas situaciones los intereses políticos frecuentemente interfieren en los intentos de la revitalización de las lenguas en peligro. Como resultado, los lingüistas muchas veces encuentran dificultades para quedarse imparciales y dedicarse solamente al estudio de la lengua. 
La cuarta regla alude a la relatividad de las normas éticas. Es importante darse cuenta de que nuestra experiencia es muy diferente de la de los miembros de las comunidades vulnerables. Por esta razón, es nuestra obligación ser comprensivos y no tomar nuestras maneras de comportamiento por universales. En este aspecto nos ayudará nuestra capacidad de observación y adaptación a la situación. Esto no significa que se deba imitar todo lo que podemos ver, sino más bien pensar más de lo habitual sobre cual puede ser nuestra conducta más adecuada en cada situación.

La última sugerencia representa probablemente el problema más grave, ya que es naturalmente deseable que la presencia del lingüista tenga también efectos positivos para la comunidad estudiada. Al pensar sobre la pregunta de qué puede ofrecer un lingüista a la comunidad estudiada llegamos a tres posibles respuestas: resultados de su trabajo, dinero y su mano de obra. Un resultado de la investigación, como un diccionario, una gramática o un manual de enseñanza, significa sin duda un aporte importante para la comunidad. Sin embargo, no todas las investigaciones tienen el potencial de generar una obra notable, y además no podemos esperar que los miembros de las comunidades vulnerables aprecien los resultados académicos.

La otra posibilidad sugerida, la recompensa económica, conlleva varios problemas que se deben considerar. Es evidente que en las comunidades específicas el investigador no puede esperar que los sujetos vayan a participar sin esperar recompensa por su tiempo y esfuerzo. Por esta razón, hace falta establecer un precio justo que motive a los participantes y que no cree un ambiente de envidia. Es recomendable consultar este asunto con los miembros escolarizados y respetados de la comunidad. La tercera manera de ayudar a la comunidad estudiada es la mano de obra que puede ofrecer el investigador. Las comunidades muchas veces necesitan ayuda en la formulación de proyectos de desarrollo, trabajos informáticos, traducciones, correcciones de textos y también con la enseñanza. En resumen, es evidente que las posibilidades del investigador para ayudar a la comunidad estudiada son limitadas. Por esta razón, la buena voluntad de ayudar tiene más valor que el propio resultado por evidenciar el respeto a la comunidad receptora.

\section{Problemas metodológicos}

La investigación en una comunidad vulnerable ocasiona también problemas metodológicos específicos que pueden variar de una comunidad a otra. Por ejemplo, el método «friend of a friend» (Milroy 1987) a veces traducido como «bola de nieve», utilizado para el reclutamiento de posibles participantes en la entrevista, funcionó de manera prevista y satisfactoria en la comunidad shuar. Sin embargo, en la comunidad lakhota fracasó rotundamente. En esta comunidad fue mucho más fácil conseguir la colaboración de una persona desconocida que la de un amigo o persona parcialmente conocida.

En cambio, una de las dificultades imprevistas que surgieron en las comunidades shuar fue la inexistencia de un lugar tranquilo apto para obtener una grabación de buena calidad. Primero fue sumamente difícil explicar a los participantes que sus hijos no podían estar presentes a la grabación y todavía más difícil explicárselo a los niños mismos, motivados por su curiosidad. Segundo, incluso sin la presencia de terceros, el ambiente raras veces era tranquilo ya que la selva misma es ruidosa por su naturaleza. En consecuencia, algunas veces no quedó otro remedio que aceptar la calidad tal como era, aunque no fuera del todo satisfactoria. 
En general, en las comunidades vulnerables el investigador se encuentra con los problemas metodológicos propios de todas las investigaciones sociolingüísticas, que no se van a discutir en este trabajo, además de los específicos a las comunidades estudiadas. Por esta razón, es necesario prever las posibles complicaciones y estar preparado a que no todas las entrevistas salen a nuestra conveniencia.

Grinevald (2003: 61) sugiere la siguiente regla general respecto a la ética de las investigaciones sociolingüísticas: «A good rule of thumb for fieldwork should be to not leave the field any worse than it was before, giving time to situations to evolve and working relations to take shape». Por lo que se refiere a la ética, parece un requerimiento bastante reducido no dejar la comunidad peor de lo que fue. Sin embargo, más importante parece la segunda parte de la cita, que se refiere también a la metodología de las investigaciones, ya que el tiempo y buenas relaciones en las comunidades receptoras son verdaderamente la clave del éxito de una investigación.

\section{Conclusión}

El tema de la ética de una investigación se vuelve especialmente sensible en las comunidades vulnerables. No obstante, la relatividad de los enfoques éticos es la causa por la que los investigadores en el campo de la sociolingüística no encuentran unos modelos del comportamiento ético claramente definidos. Otro aspecto que dificulta la aplicación de los procedimientos estándares es la incapacidad de los miembros de estas comunidades de tomar una decisión verdaderamente informada, considerando todos los aspectos de su participación. El campo de la metodología también aporta problemas específicos al trabajar con las comunidades vulnerables. A este respecto es crucial tener una buena relación con la comunidad que asegure su colaboración y sobre todo mucha paciencia.

El objetivo de este trabajo fue el de llamar la atención sobre algunos temas que deben considerarse al trabajar con las comunidades vulnerables. No se trata de unas reglas que cubren de manera exhaustiva la ética de las investigaciones, más bien son unos puntos importantes que no se pueden descuidar al emprender una investigación sociolingüística. Aunque el investigador posiblemente no sea capaz de cumplir con todos los requisitos formales, el sentido común y el respeto hacia la comunidad deberían guiarle en su comportamiento.

En resumen, el tema de la ética es bastante complejo y a veces descuidado en relación con las investigaciones lingüísticas. Al tratar las comunidades vulnerables es conveniente tomar en cuenta las reglas mencionadas anteriormente, sin embargo, lo más importante consiste en pensar mucho sobre la ética de nuestra investigación y en no tomar por sobreentendido que todo está en orden. Solo de esta manera podemos asegurar el trato justo y respetuoso a todos los participantes de nuestra investigación.

\section{Bibliografía}

GrineVALD, Colette (2003), «Speakers and documentation of endangered languages», in: Austin, Peter K. (ed.), Language documentation and description, vol.1, London: SOAS, 52-72.

Johnstone, Barbara (2000), Qualitative methods in sociolinguistics, New York, Oxford: Oxford University Press. 
Milroy, Lesley (1987), Language and social networks, Oxford: Blackwell.

Valeš, Miroslav (2010), Observaciones sociolingüísticas del español, Saarbrücken: Lambert Academic Publishing.

Miroslav Valeš

Katedra románských jazyků

Fakulta přírodovědně-humanitní

a pedagogická

Technická univerzita v Liberci

Studentská 2

46117 Liberec

República Checa

miroslav.vales@tul.cz 